\title{
Prospección de acuíferos con métodos electromagnéticos en la ciudad de Ávila, España
}

\author{
Prospecting aquifers with electromagnetic methods \\ in the city of Ávila, Spain
}

\author{
Arturo Santos Zevallos Flores ${ }^{1}$, Pedro Carrasco García ${ }^{2}$
}

Recibido: 01/07/2020 - Aprobado: 30/10/2020 - Publicado: 30/11/2020

\begin{abstract}
RESUMEN
El municipio de Casas del Puerto se ubica SW de la ciudad de Ávila, actualmente no cuenta con agua suficiente para consumo humano y riego, por lo que el agua subterránea representa una gran posibilidad de contar con el recurso hídrico, para ello se utilizara un Método Electromagnético Very Low Frecuency VLF. Las medidas de las longitudes de los perfiles deben ser lo suficientemente largos, al menos para abarcar la anomalía total.

El articulo tiene como objetivo determinar las posibilidades hidrogeológicas de los materiales que componen el área de investigación en el Municipio de Casas del Puerto, Ciudad de Ávila, la cual tiene como parcelas (1-18-31), los polígonos (3-4-6), con un área total de 10.844 hectáreas, así mismo determinar la conductividad de los cuerpos, para luego caracterizarlo litológica e hidrogeológicamente y finalmente determinar las zonas más favorables para emplazar sondeos de captación de aguas subterráneas.
\end{abstract}

Con la Interpretación geológica y un análisis de la Componente real (ángulo de inclinación) e imaginaria (elipticidad), se logró diferenciar dos zonas con menor posibilidad hidrogeológica, por lo cual la Zona-3, presenta un gran contraste de los cuerpos conductores, debido a la zona de contacto.

Palabras clave: Agua subterránea; geofísica; posibilidad de captar aguas subterráneas.

\begin{abstract}
The municipality of Casas del Puerto is located SW of the city of Ávila, currently it does not have enough water for human consumption and irrigation, so groundwater represents a great possibility of having the water resource, for which a method Very Low Frequency VLF Electromagnetic. Profile length measurements should be long enough, at least to encompass the total anomaly.

The article aims to determine the hydrogeological possibilities of the materials that make up the research area in the Municipality of Casas del Puerto, Ciudad de Ávila, which has plots (1-18-31), polygons (3-4-6), with a total area of 10,844 hectares, likewise determine the conductivity of the bodies, to later characterize it lithologically and hydrogeologically and finally determine the most favorable areas to place groundwater collection wells.

With the geological Interpretation and an analysis of the real Component (angle of inclination) and imaginary (ellipticity), it was possible to differentiate two zones with less hydrogeological possibility, therefore Zone-3 presents a great contrast of the conductive bodies, due to the contact area.
\end{abstract}

Keywords: Underground water; geophysics; possibility of capturing groundwater.

\footnotetext{
1 Licenciado Físico de la Universidad Nacional Mayor de San Marcos, Facultad de Ciencias Físicas. Lima, Perú. Autor para correspondencia: arturo.zevallos@unmsm.edu.pe ORCID: https://orcid.org/0000-0003-0033-2083

2 Docente del Área de Prospección e Investigación Minera, Escuela Politécnica Superior de Ávila, Calle Hornos Caleros, 50; 05003. Ávila, España. E-mail: retep81@usal.es
} 


\section{INTRODUCCIÓN}

El articulo tiene como objetivo determinar las posibilidades hidrogeológicas de los materiales que componen el área de investigación en el Municipio de Casas del Puerto, Ciudad de Ávila, la cual tiene como parcelas (1-18-31), los polígonos (3-4-6), con un área total de 10.844 hectáreas; a partir de ello, se determinará la conductividad de los cuerpos, para luego caracterizarlo litológica e hidrogeológicamente y finalmente determinar las zonas más favorables para emplazar sondeos de captación de aguas subterráneas.

Para la investigación se aplicara el Método Electromagnético Very Low Frecuency VLF, basado en el análisis de las modificaciones que generan en las propiedades eléctricas del subsuelo las ondas de radio de baja frecuencia según (Kaikkonen \& Sharma, 1997). Así mismo el método VLF hace uso de las componentes magnéticas de los campos electromagnéticos generados por trasmisoras militares de frecuencias de radio que generalmente usan el ancho de banda llamado VLF (Very Low Frecuency) de 3 a $30 \mathrm{kHz}$. Las fuentes trasmisoras pueden ser detectadas a miles de kilómetros, y que son utilizadas para la prospección geofísica de zonas fracturadas, agua subterránea, contaminantes y minerales según (Jeng et al., 2004), (Drahor, 2006), (Kaya et al., 2007). La existencia de fallas y fracturas en un medio hidrogeológico contribuye a mejorar la conductividad hidráulica y su porosidad, y actúan a su vez como estructuras de control del movimiento del agua subterránea según (Sharma \& Baranwal, 2005), (Adepelumi et al., 2006). Esto a su vez ocasiona perturbaciones en las señales medidas con instrumentos VLF.

La investigación se ha realizado utilizando el instrumento ABEM WADI. La frecuencia de recepción se ha establecido en $19.58 \mathrm{kHz}$ (GBZ, Tabla 2).
Para la localización de fracturas y aprovechamiento hidrogeológico en el T.M de Casas del Puerto de Villatoro (Ávila) (Figura 1), se realizaron una serie de perfiles VLF a estos perfiles de investigación se aplicó el filtro (Karous \& Hjelt, 1983), para obtener la sección de densidad de corriente. El perfil se realizó tomando medidas cada 5 y $10 \mathrm{~m}$ para la detección de fracturas en granitos, para posteriormente instalar sondeos de aprovechamiento de acuerdo (Carrasco García, 2011).

La justificación del método VLF de aplicación en este caso es muy simple. Hay un gran contraste de resistividad entre las capas que se producen allí. Las arcillas y margas tienen una resistividad eléctrica muy baja (Tabla 3), pero las arenas (incluso saturadas de agua) se caracterizan por una resistividad más alta (Tabla 4). Las resistividades de diferentes rocas varían dentro de amplios límites. El agua salina, que satura rocas, arcillas y arenas, se caracteriza por la conducción iónica, y sus resistividades son relativamente bajas (Antoniuk et al., n.d.). El aumento de la salinidad del agua provoca un aumento significativo en su conductividad (Plewa \& Plewa, 1992).

\subsection{Ubicación y acceso del área de investigación}

El área de investigación, se encuentra en el municipio de Casas del Puerto de Villatoro (Ávila), lugar donde se encuentran las parcelas (1-18-31) de los polígonos (3-4-6) y con un área total de 10.844 hectáreas, las coordenadas de los perfiles de VLF son:

Para hacer los trabajos de campo, iniciamos el recorrido desde Madrid llegando al Municipio de Casas del Puerto de Villatoro en 100 minutos, recorriendo una distancia de $150 \mathrm{~km}$; luego nos dirigimos con un guía al área de investigación, mediante una carretera afirmada. En el Término Municipal de Casas del Puerto de Villatoro la actividad principal es la agricultura (Ver Tabla 1 y Figura 2).

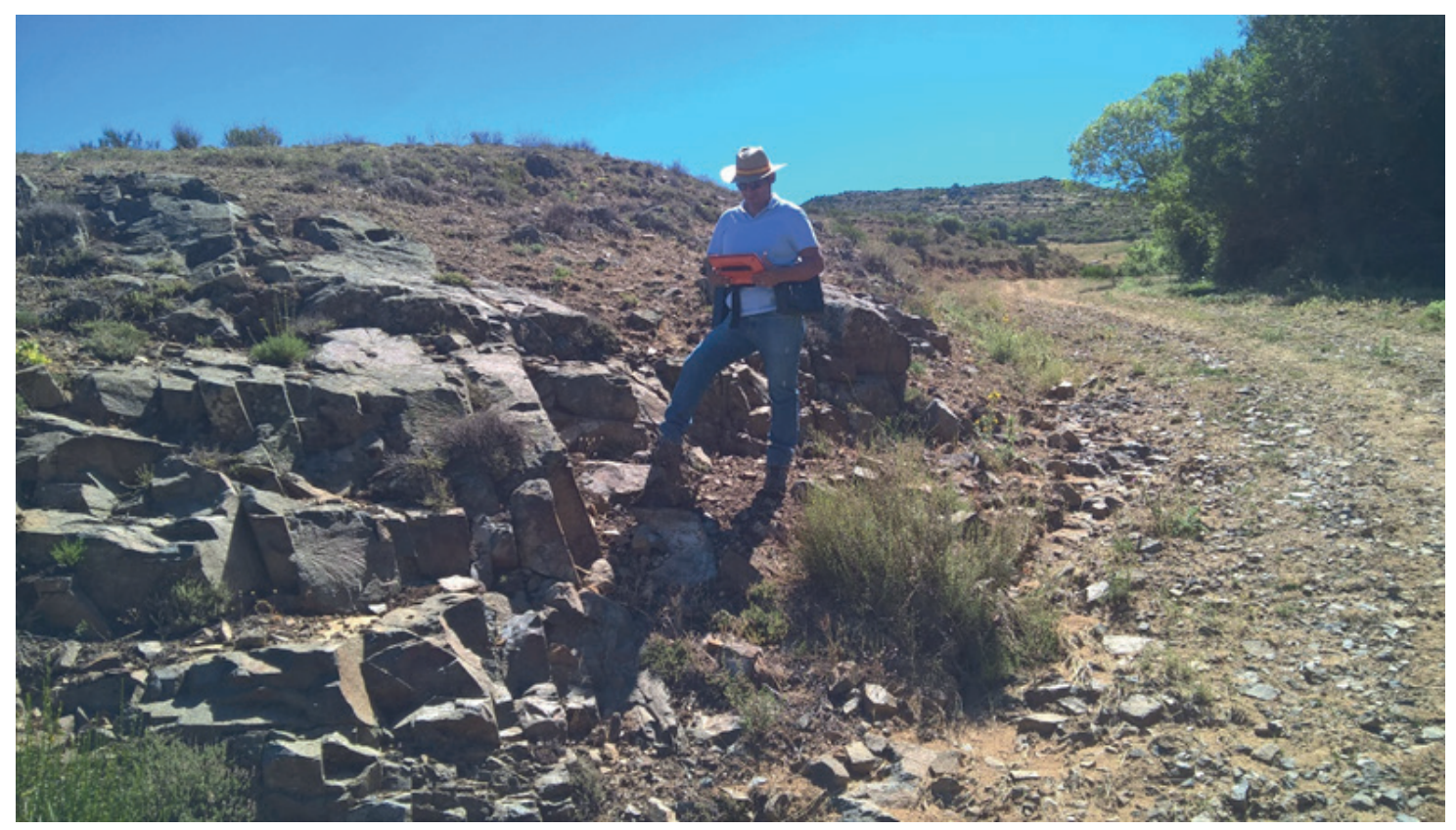

Figura 1. Tomando medidas de VLF cada 10m, tercera zona de estudio.

Fuente: Elaboración Propia 
Tabla 1. Coordenadas de los Perfiles de VLF

\begin{tabular}{lcccccc}
\hline Perfiles & \multicolumn{3}{c}{ Inicio } & & \multicolumn{2}{c}{ Final } \\
& Este ETRS89 & Norte ETRS89 & Cota msnm & Este ETRS89 & Norte ETRS89 & Cota msnm \\
\hline VLF-1 & 313119.00 & 4488010.00 & 1305.00 & 313198.00 & 4487915.00 & 1306.00 \\
VLF-2 & 313647.00 & 4488241.00 & 1224.00 & 313683.00 & 4488443.00 & 1223.00 \\
VLF-3 & 314801.00 & 4488141.00 & 1213.00 & 314618.00 & 4488241.00 & 1212.00 \\
\hline
\end{tabular}

Fuente: Elaboración Propia
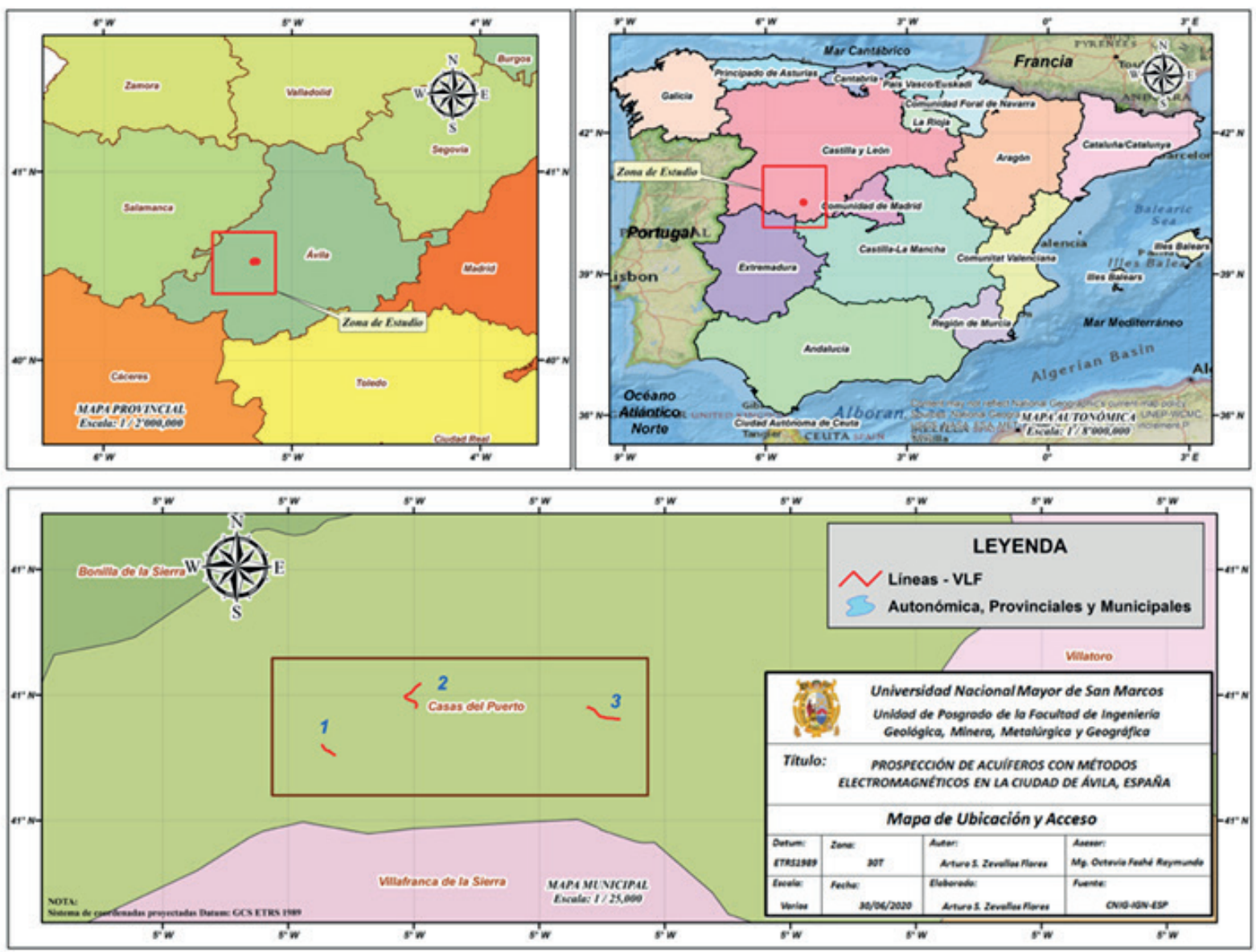

Figura 2. Ubicación del Área de Investigación. Municipios - Provincias y C. Autónomas. Fuente: Elaboración Propia

\subsection{Geología Regional}

\section{Situación del Perfil VLF - 1}

Geológicamente, la primera área de estudio se encuadra dentro del Terciario - Neógeno, constituidos por arcillas, limos - arenas (aluvial-coluvial) y diques de leucogranitos aplíticos biotíticos con moscovita y cordierita (Granodiorita alterado) (Hojas N529 Santa María del Berrocal) - IGME.

\section{Situación del Perfil VLF - 2}

Geológicamente, la segunda zona de estudio se encuentra constituida por diques de cuarzo y diques de leucogranitos aplíticos biotíticos con moscovita y cordierita (Granodiorita alterado) (Hojas N529 Santa María del Berrocal) - IGME.

\section{Situación del Perfil VLF - 3}

Geológicamente, la tercera zona de estudio se encuentra constituida por un dique de diabasa (Alentejo - Plasencia), diques de leucogranitos aplíticos biotíticos con moscovita y cordierita (Granodiorita alterado) y conglomerados, areniscas y cuarcitas (Hojas N529 Santa María del Berrocal y N530 Vadillo de La Sierra) - IGME.

\subsection{Objetivo de la Investigación}

Determinar las posibilidades hidrogeológicas de los materiales que componen el área de investigación en el Municipio de Casas del Puerto, Ciudad de Ávila, la cual tiene como parcelas (1-18-31), los polígonos (3-4-6), con un área total de 10.844 hectáreas. 
1. Determinar la conductividad de los cuerpos mediante la interpretación de la amplitud, ángulo de inclinación y las anomalías de elipticidad en los Perfiles Electromagnéticos VLF.

2. Caracterizar litológica e hidrogeológicamente las unidades geoeléctricas existentes en la zona de estudio y detectadas con la prospección geofísica.

3. Determinar las zonas más favorables para emplazar sondeos de captación de aguas subterráneas (Ver Figura 3).

\section{METODOLOGÍA}

El método consiste en la investigación de los campos secundarios generados por cuerpos conductores en profundidad, que se inician desde un campo EM primario. La fuente del VLF se considera artificial y pasiva, ya que proviene de una transmisión de señales de radio militares $\mathrm{u}$ otros, con miles de $\mathrm{km}$ de distancia, es por ello que las ondas EM, se consideran plana (Tabla 2). Según (Kaufman \& Keller, 1981), indica que se deben realizar secciones adicionales para tener un mayor alcance de estos cuerpos conductores en la zona de estudio, ya que método por teoría se aplica la investigación de las secciones de forma ortogonal a la estructura.
Las componentes medidas se puede dividir: en modo magnético (Proviene del Secundario $-\mathrm{H}_{\mathrm{Z}}$ y $\mathrm{H}_{\mathrm{Y}}$ ) y eléctrico (Proviene del campo Primario y Secundario - $\mathrm{E}_{\mathrm{X}} \mathrm{y} \mathrm{H}_{\mathrm{Y}}$ ). La resistividad aparente, se calcula apartir de la impedancia $\left(\mathrm{Z}=\mathrm{E}_{\mathrm{X}} / \mathrm{H}_{\mathrm{Y}}\right)$, y de ecuación de Zhdanov \& Keller (1994), obtenemos:

$$
\rho_{\mathrm{a}}=\frac{1}{\omega \mu_{0}} *\left|\frac{\mathrm{E}_{\mathrm{X}}}{\mathrm{H}_{\mathrm{Y}}}\right|^{2}
$$

Tenemos que: $\rho_{\mathrm{a}}$ : resistividad $(\Omega \mathrm{m}), \omega=2 \pi \mathrm{f}$ Frecuencia Angular (f en hz), $\mu_{0}=4 * 10^{-7}$ permeabilidad

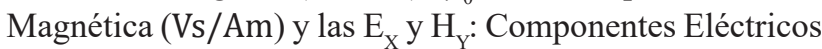
$(\mathrm{mV} / \mathrm{m})$ y magnéticos y $(\mu \mathrm{A} / \mathrm{m})$.

En el suelo, el campo magnético se altera en fase y dirección. Está atenuado, y el rango de profundidad está determinado por el efecto Skin. Según Spies (1989), el rango de profundidad se puede estimar:

$$
\delta=503.3 \sqrt{\rho / \mathrm{f}}
$$

Donde $\delta$ : profundidad del Skin $(\mathrm{m}), \mathrm{f}$ : frecuencia $(\mathrm{Hz})$ de la fuente y $\rho_{\mathrm{a}}$ la resistividad $\left(\Omega^{*} \mathrm{~m}\right)$ del medio (Tabla 3 ).

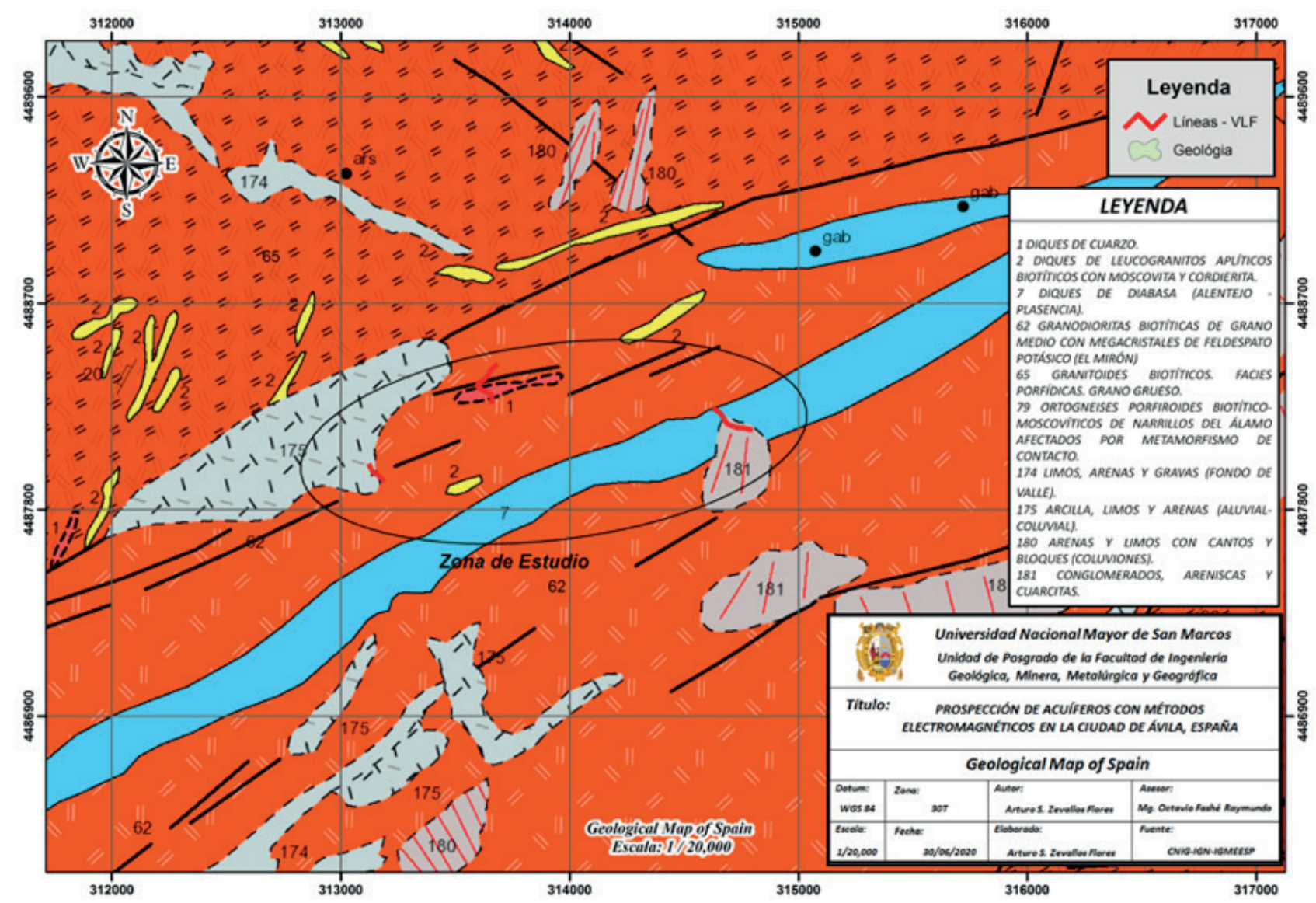

Figura 3. Mapa Geológico (Hojas 529 - Sta. María del Berrocal y 530 - Vadillo de La Sierra) - IGME. Fuente: Elaboración Propia 
Tabla 2. Lista de Transmisores VLF disponibles

\begin{tabular}{|c|c|c|c|c|c|}
\hline Señal & Frequencia & Potencia & Posición & Latitud & Longitud \\
\hline \multirow{2}{*}{ GBZ } & \multirow{2}{*}{19580} & \multirow{2}{*}{$500 \mathrm{~kW}$} & \multirow{2}{*}{ Anthorn, UK Locator: IO84iv } & N $54^{\circ} 54^{\prime} 41.91^{\prime \prime}$ & W $003^{\circ} 16^{\prime} 42.44^{\prime \prime}$ \\
\hline & & & & $\left(+54.911643^{\circ}\right)$ & $\left(-003.278456^{\circ}\right)$ \\
\hline \multirow{2}{*}{ ICV } & \multirow{2}{*}{20270} & \multirow{2}{*}{ 20/43kW } & \multirow{2}{*}{ Isola di Tavolara, Italy Locator: JN40uw } & $N 40^{\circ} 55^{\prime} 23.26^{\prime \prime}$ & E $009^{\circ} 43^{\prime} 51.64^{\prime \prime}$ \\
\hline & & & & $\left(+40.923127^{\circ}\right)$ & $\left(+009.731011^{\circ}\right)$ \\
\hline \multirow{2}{*}{ GQD } & \multirow{2}{*}{22100} & \multirow{2}{*}{$500 \mathrm{~kW}$} & \multirow{2}{*}{ Skelton, UK Locator: IO84nr } & N $54^{\circ} 43^{\prime} 54.48^{\prime \prime}$ & W $002^{\circ} 52^{\prime} 58.92^{\prime \prime}$ \\
\hline & & & & $\left(+54.731799^{\circ}\right)$ & $\left(-002.883033^{\circ}\right)$ \\
\hline \multirow{2}{*}{ DHO38 } & \multirow{2}{*}{23400} & \multirow{2}{*}{$800 \mathrm{~kW}$} & \multirow{2}{*}{ Rhauderfehn, Germany Locator: JO33tb } & N $53^{\circ} 04^{\prime} 44.04^{\prime \prime}$ & $\mathrm{E} 007^{\circ} 36^{\prime} 54.00^{\prime \prime}$ \\
\hline & & & & $\left(+53.078900^{\circ}\right)$ & $\left(+007.615000^{\circ}\right)$ \\
\hline \multirow{2}{*}{ NAA } & \multirow{2}{*}{24000} & \multirow{2}{*}{$1000 \mathrm{~kW}$} & \multirow{2}{*}{ Cutler, ME, USA: Locator: FN64ip } & $\mathrm{N} 44^{\circ} 38^{\prime} 41.77^{\prime \prime}$ & W $067^{\circ} 16^{\prime} 53.90^{\prime \prime}$ \\
\hline & & & & $\left(+44.644936^{\circ}\right)$ & $\left(-067.281639^{\circ}\right)$ \\
\hline \multirow{2}{*}{ NLK } & \multirow{2}{*}{24800} & \multirow{2}{*}{$250 \mathrm{~kW}$} & \multirow{2}{*}{ Oso Wash, Jim Creek, WA - USA. Locator: CN98ae } & N $48^{\circ} 12^{\prime} 12.55^{\prime \prime}$ & W $121^{\circ} 55^{\prime} 0.58^{\prime \prime}$ \\
\hline & & & & $\left(+48.203487^{\circ}\right)$ & $\left(-121.916827^{\circ}\right)$ \\
\hline \multirow{2}{*}{ NML } & \multirow{2}{*}{25200} & \multirow{2}{*}{$500 \mathrm{~kW}$} & \multirow{2}{*}{ La Moure, ND Locator, USA: EN06ti } & N $46^{\circ} 21^{\prime} 57.56^{\prime \prime}$ & W $098^{\circ} 20^{\prime} 8.30^{\prime \prime}$ \\
\hline & & & & $\left(+46.365990^{\circ}\right)$ & $\left(-098.335638^{\circ}\right)$ \\
\hline \multirow{2}{*}{ TBB } & \multirow{2}{*}{26700} & & \multirow{2}{*}{ Bafa, Turkey Locator: KM37pj } & N $37^{\circ} 24^{\prime} 45.81^{\prime \prime}$ & E $027^{\circ} 19^{\prime} 24.03^{\prime \prime}$ \\
\hline & & & & $\left(+37.412725^{\circ}\right)$ & $\left(+027.323342^{\circ}\right)$ \\
\hline \multirow{2}{*}{ NRK/TFK } & \multirow{2}{*}{37500} & \multirow{2}{*}{$100 \mathrm{~kW}$} & \multirow{2}{*}{ Grindavik, Iceland Locator: HP83su } & $N 63^{\circ} 51^{\prime} 1.31^{\prime \prime}$ & W $022^{\circ} 28^{\prime} 0.38^{\prime \prime}$ \\
\hline & & & & $\left(+63.850365^{\circ}\right)$ & $\left(-022.466773^{\circ}\right)$ \\
\hline \multirow{2}{*}{ NAU } & 40800 & $100 \mathrm{~kW}$ & Aquada Puerto Rico I ncator. FK68ii & N $18^{\circ} 23^{\prime} 55.54^{\prime \prime}$ & W $067^{\circ} 10^{\prime} 39.36^{\prime \prime}$ \\
\hline & & & 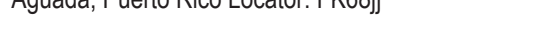 & $\left(+18.398762^{\circ}\right)$ & $\left(-067.177599^{\circ}\right)$ \\
\hline
\end{tabular}

Fuente: GEM Systems (2008)

Tabla 3. Valores de resistividad $(\rho)$ y conductividad $(\sigma)$ de rocas (Kobranova, 1990)

\begin{tabular}{lcc}
\hline Rock & $\rho[\Omega \mathrm{m}]$ & $\sigma[\mathrm{mS} / \mathrm{m}]$ \\
\hline Arena & $100-2500$ & $0.4-10$ \\
Arcilla & $1-100$ & $10-1000$ \\
Marga & $5-50$ & $20-200$ \\
Margas & $3-70$ & $14-300$ \\
Arenisca & $500-5000$ & $2-20$ \\
Caliza & $1-2 \times 10^{5}$ & $0.5-0.01$ \\
Yeso & $10^{5}-10^{6}$ & $0.01-0.001$ \\
Anhidrita & $10^{2}-10^{5}$ & $0.001-10$ \\
Sal de roca & $10^{4}-10^{8}$ & $10^{-5}-0.1$ \\
\hline
\end{tabular}

Tabla 4. Valores de resistividad $(\rho)$ y conductividad $(\sigma)$ de areniscas (Guinea et al., 2010)

\begin{tabular}{ccc}
\hline Material & $\rho[\Omega \mathrm{m}]$ & $\sigma[\mathrm{mS} / \mathrm{m}]$ \\
\hline Arenisca Sólida & $>1000$ & $<1$ \\
Arenisca Saturada & $<100$ & $<10$ \\
\hline
\end{tabular}

Para la toma de datos del método VLF se usó un equipo ABEM WADI realizando tres perfiles en las diferentes zonas de estudio. La longitud de los perfiles fue de 130 , 270 y $220 \mathrm{~m}$ y la separación entre estaciones de 5 y $10 \mathrm{~m}$.

Los datos del estudio VLF (Tabla 5) se analizan para la explotación de aguas subterráneas, durante el procesamiento de los datos de campo VLF-EM se le aplicó el filtro (Karous \& Hjelt, 1983) obteniendo datos de mejor calidad, utilizando el Software RAMAG v2.2 (ABEM Instrument $\mathrm{AB}, 1989)$.

Luego del procesamiento se obtiene los datos filtrados, (Figuras 4, 5, 6, 7, 8 y 9) las pseudo secciones de densidad de corriente para cada perfil, ubicación de la anomalía y profundidad aproximada en cada perfil, estas informaciones son extraídas de los gráficos para realizar una interpretación y evaluación en la prospección de acuíferos. Las observaciones muestran que hay varias anomalías con diversas intensidades de densidad de corriente, que varían con la profundidad.

\section{RESULTADOS}

El resultado en el procesado e interpretación de los perfiles y secciones ha tenido como objetivo principal la determinación de los cambios de conductividad, Zonas de fracturas y diques con la finalidad de establecer los cambios laterales y las unidades geoléctricas. Finalmente seleccionar las zonas más favorables para el emplazamiento de sondeos y la captación de aguas subterráneas.

Con lo que se resaltaron las zonas fracturadas asociadas con la posibilidad que contenga con agua y otros contrastes de conductividad en el subsuelo, presentándose en secciones de densidad de corriente donde los valores intermedios en porcentaje representan zonas de contacto, de hasta 10\%. El filtrado depende de la separación de las estaciones medidas, aquí se usó una profundidad de filtrado de $30 \mathrm{~m}$. 
Tabla 5. Partes Reales e Imaginarias de datos de lectura VLF

\begin{tabular}{|c|c|c|c|c|c|c|c|c|}
\hline \multicolumn{3}{|c|}{ 0100E - VLF1 } & \multicolumn{3}{|c|}{ 0101E-VLF2 } & \multicolumn{3}{|c|}{ 0102E-VLF3 } \\
\hline Station & Real parts & $\begin{array}{c}\text { Imaginary } \\
\text { parts }\end{array}$ & Station & Real parts & $\begin{array}{c}\text { Imaginary } \\
\text { parts }\end{array}$ & Station & Real parts & $\begin{array}{c}\text { Imaginary } \\
\text { parts }\end{array}$ \\
\hline 0 & 14 & 0.4 & 0 & -21.7 & -1 & 0 & -3.2 & 5.7 \\
\hline 5 & 15.3 & 9.8 & 10 & -26.9 & -12.8 & 10 & -8.1 & 6.7 \\
\hline 10 & 7.7 & 7.2 & 20 & -27.5 & -6.6 & 20 & -13.3 & 7.6 \\
\hline 15 & 12.2 & -4.9 & 30 & -31.8 & 1.3 & 30 & -18.9 & 7.6 \\
\hline 20 & 10.5 & 10.1 & 40 & -22.3 & -5.2 & 40 & -22 & 7.9 \\
\hline 25 & 5.2 & 6.5 & 50 & -21.9 & -5.7 & 50 & -23.4 & 8.1 \\
\hline 30 & 17.7 & 16.4 & 60 & -16.8 & -20.4 & 60 & -27.4 & 8.5 \\
\hline 35 & 13.9 & -0.7 & 70 & -7.1 & -8.2 & 70 & -28.8 & 9.2 \\
\hline 40 & -4.5 & 17.1 & 80 & -4.3 & -6.9 & 80 & -36.4 & 10.9 \\
\hline 45 & -3.2 & 13.2 & 90 & 0 & -18.2 & 90 & -45.1 & 10.8 \\
\hline 50 & 3.9 & 9.6 & 100 & -8.6 & -11.7 & 100 & -56.2 & 10.8 \\
\hline 55 & 3 & 6.2 & 110 & -16 & -17.4 & 110 & -69.9 & 8.5 \\
\hline 60 & 3.9 & -0.6 & 120 & -7.8 & -2.5 & 120 & -63.1 & 6.2 \\
\hline 65 & 4.7 & 8.1 & 130 & 9.3 & 1.1 & 130 & -39.6 & 8.4 \\
\hline 70 & -1.9 & 12.2 & 140 & 4.9 & -8.1 & 140 & -37.3 & 5.7 \\
\hline 75 & 5.1 & 2.4 & 150 & 22.2 & 1.6 & 150 & -39.6 & 5.4 \\
\hline 80 & 6.9 & 4.9 & 160 & 18.5 & -1.2 & 160 & -42.3 & 6.8 \\
\hline 85 & 0.9 & 10 & 170 & -1.4 & 6.2 & 170 & -35.5 & 0.2 \\
\hline 90 & -3.1 & 0.2 & 180 & 1 & -0.9 & 180 & -25.8 & -2.7 \\
\hline 95 & -3.9 & 9.1 & 190 & 18.1 & -2.9 & 190 & -24.1 & -8 \\
\hline 100 & 1.5 & 9.2 & 200 & 11 & 2.6 & 200 & -9.2 & -10.3 \\
\hline 105 & -6.6 & -3.3 & 210 & 1.3 & -3.4 & 210 & -0.1 & -9.3 \\
\hline 110 & -0.9 & 0.2 & 220 & 8.5 & 0 & 220 & 12.1 & -9.8 \\
\hline 115 & -19.9 & 6.4 & 230 & 18.6 & 3.6 & & & \\
\hline 120 & -5.7 & 6.9 & 240 & 9.5 & -3.3 & & & \\
\hline 125 & -5.5 & -3.8 & 250 & 10.5 & 2.1 & & & \\
\hline \multirow[t]{2}{*}{130} & -17.1 & 3.1 & 260 & -0.6 & 5.9 & & & \\
\hline & & & 270 & -82.5 & 29.3 & & & \\
\hline
\end{tabular}

Fuente: Elaboración Propia

En la Figura 4. Se muestra un perfil filtrado para la primera zona. Se observa que la componente en fase (real) es mayor que la componente en cuadratura (imaginaria) hasta la estación $108 \mathrm{~m}$, luego las componentes se invierten. Se muestran picos de anomalías conductoras pequeñas y con una anchura mediana en el gráfico de datos filtrados en fase a lo largo del Perfil VLF-1, caracterizada por menos del $10 \%$ de la densidad de corriente en la pseudo sección de corriente de filtrado (Karous \& Hjelt, 1983). El perfil indica zonas de materiales alterados y poco favorables para la presencia de agua subterránea.

La Figura 5. Muestra que la componente en fase (real) es menor que la componente en cuadratura (imaginaria) hasta la estación $165 \mathrm{~m}$, luego las componentes se invierten. Se reconoce en el perfil VLF-2. Se muestra anomalías con picos pequeños de cuerpos que son malos conductores y con una anchura mediana, así mismo presenta densidades de corriente superiores al 10\%, hasta una anomalía que se produce en el perfil, resultado de un suministro de energía durante el proceso de estudio.
La Figura 6. Muestra que la componente en fase (real) es mayor que la componente en cuadratura (imaginaria) hasta la estación $110 \mathrm{~m}$ luego las componentes se invierten. Se muestran picos máximos con anomalías conductoras y con anchura definida en el gráfico de datos filtrados en fase a lo largo del Perfil VLF-3, caracterizada por un cambio de contraste del $10 \%$ de la densidad en la pseudo sección de corriente de filtrado (Karous \& Hjelt, 1983). El perfil indica zonas de contacto y alta presencia de localizar agua subterránea.

\section{DISCUSIÓN}

Los perfiles de VLF se llevaron a cabo para determinar las posibilidades hidrogeológicas de los materiales que componen la zona de estudio. De los datos obtenidos, nuestro objetivo se identificó debido a la desviación en el grado de conductividad (componente imaginaria) y la geometría del cuerpo (componente real). 
La primera zona de estudio se ubica en dirección SurOeste donde abundan afloramientos de rocas constituidas por un granito alterado, así como la formación con niveles de cuarzos debido a los efectos del intemperismo, por lo que el uso del método VLF fue de gran utilidad. En la Figura 7 se presenta la sección de la parte real (en fase) del campo secundario mostrándose valores de densidad de corriente de hasta $30 \%$ que corresponde a zonas de material alterado.
La segunda zona de estudio se ubica en dirección SurOeste donde abundan afloramientos de rocas constituidas por un granito alterado, así como zonas conformados por niveles de cuarzos debido a los efectos del intemperismo, por lo que el uso del método VLF fue de gran utilidad. En la Figura 8 se presenta la sección de la parte real (en fase) del campo secundario mostrándose valores de densidad de corriente de hasta $30 \%$ que corresponde a una zona energizada.

\section{Perfil VLF-1}

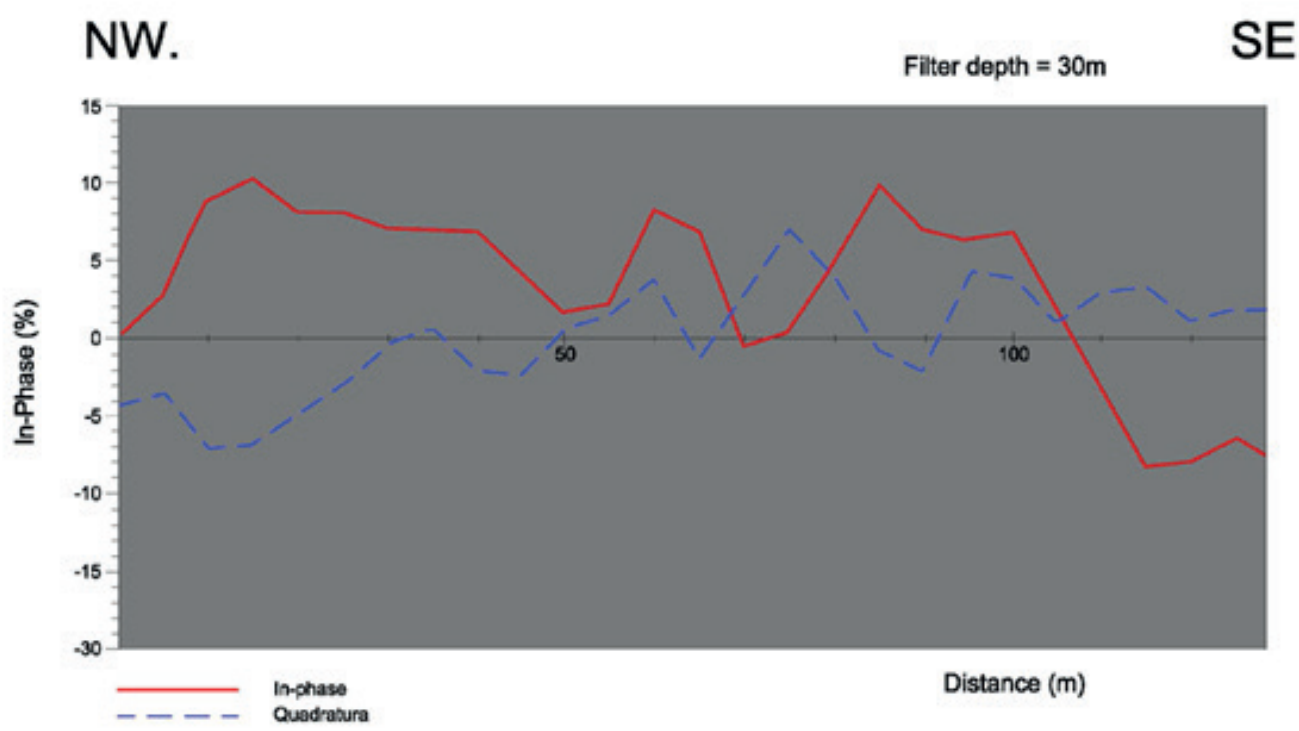

Figura 4. Componente parte real (ángulo de inclinación) y parte imaginaria (elipticidad), la primera es mayor que el segundo. El cambio en las componentes estación 108m se asocia a zonas alteradas. Fuente: Elaboración propia.

\section{Perfil VLF-2}

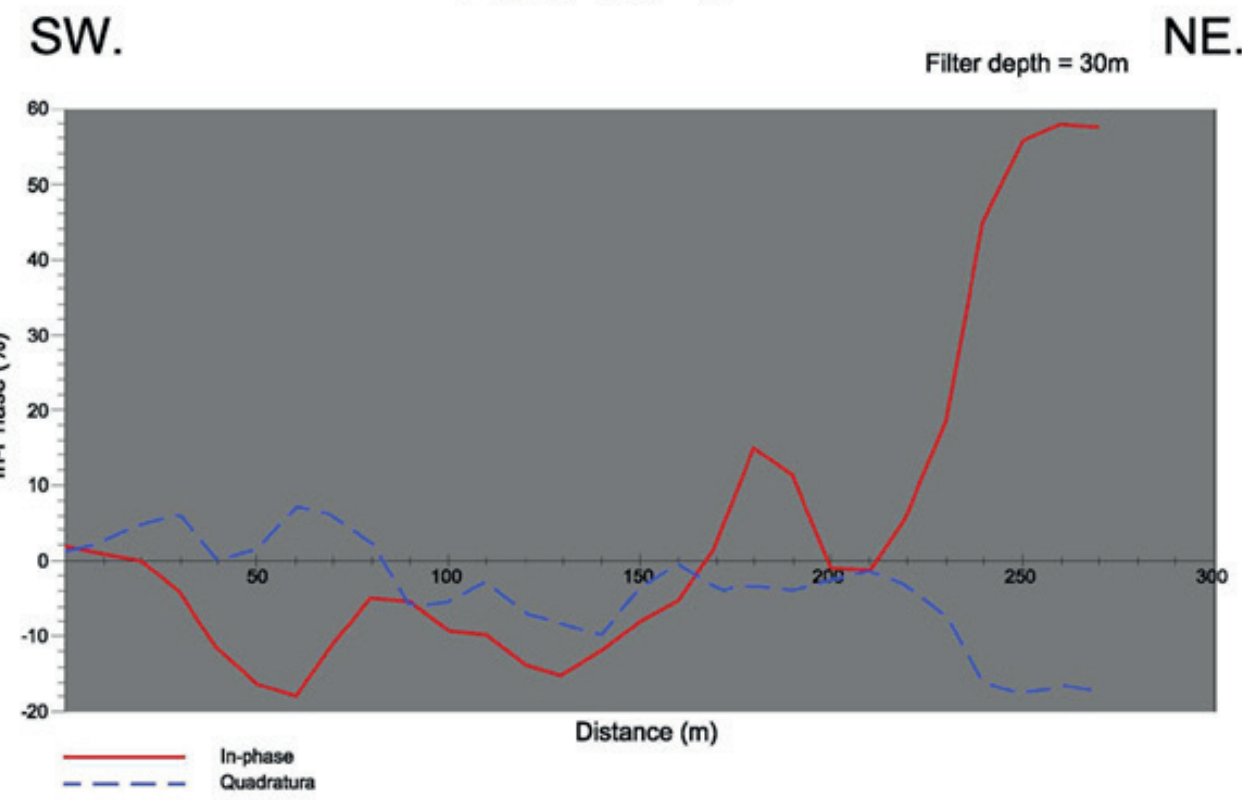

Figura 5. Componente parte real (ángulo de inclinación) y parte imaginaria (elipticidad), la segunda es mayor que el primero. El cambio en las componentes estación 165m se asocia a una línea con energía eléctrica. Fuente: Elaboración propia. 


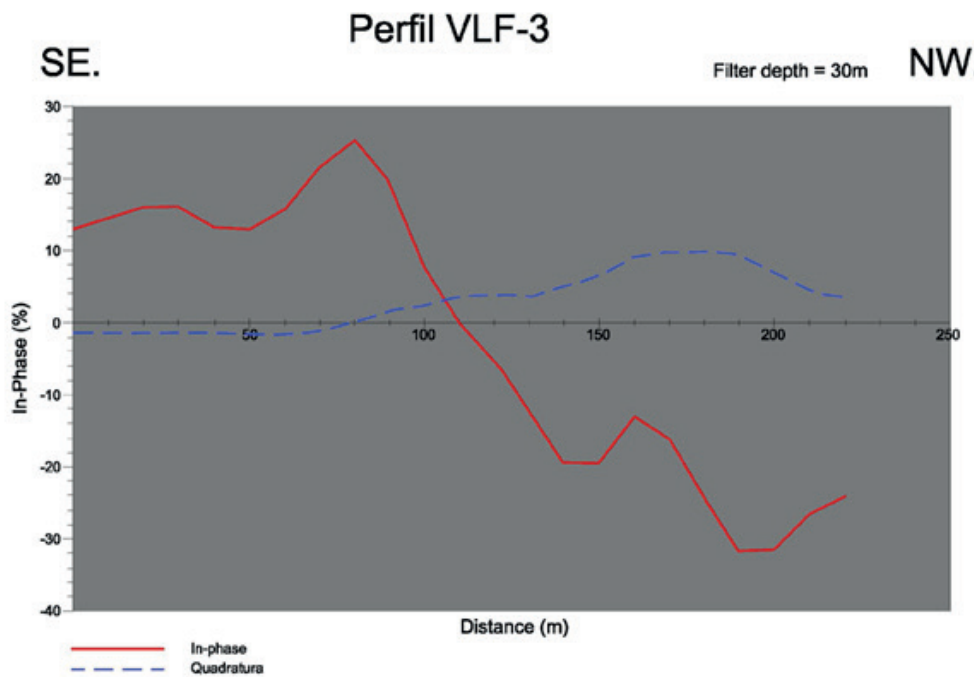

Figura 6. Componente parte real (ángulo de inclinación) y parte imaginaria (elipticidad), la primera es mayor que el segundo. El cambio en las componentes estación $110 \mathrm{~m}$ se asocia a zonas de contacto entre ambos materiales existentes, así mismo la alta posibilidad que contenga agua subterránea. Fuente: Elaboración propia.

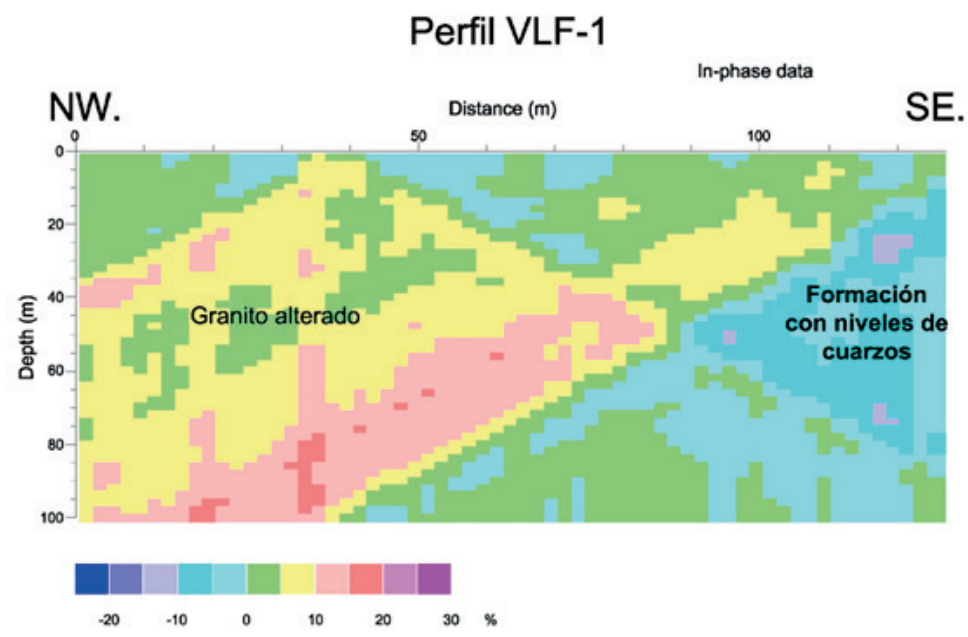

Figura 7. Sección de la parte real (en fase) de un perfil medido en la parte SW. de la ciudad de Ávila. (La zona de color magenta se asocia con altos porcentajes de densidad de corriente, relacionado con zonas alteradas. Fuente: Elaboración propia.

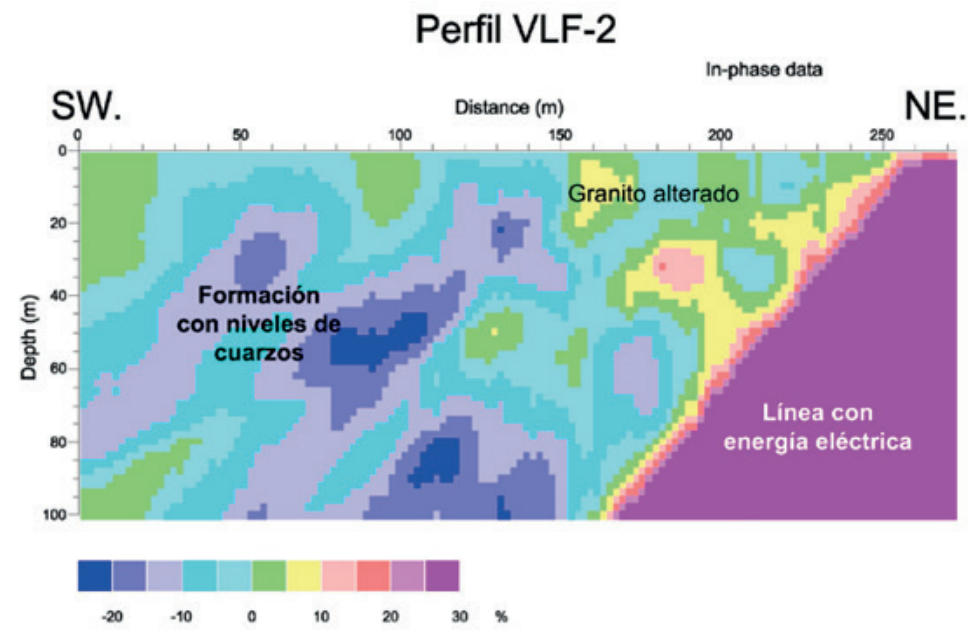

Figura 8. Sección de la parte real (en fase) de un perfil medido en la parte SW. de la ciudad de Ávila. (La zona de color magenta se asocia con altos porcentajes de densidad de corriente, relacionado con una zona energizada. Fuente: Elaboración propia. 
La tercera zona de estudio se ubica en dirección SurOeste donde abundan afloramientos de rocas constituidas por un granito alterado, así como diques de diabasa debido a los contactos de los materiales existentes presentan un índice alto de captar aguas subterráneas, por lo que el uso del método VLF fue de gran utilidad. En la Figura 9 se presenta la sección de la parte real (en fase) del campo secundario mostrándose valores de densidad de corriente intermedio de hasta $10 \%$ que corresponden a la zona de contacto de ambos materiales.

En esta misma zona de estudio se pudo identificar con el perfil VLF-3 y la geología local, las mayores posibilidades de captar aguas subterráneas con una profundidad de penetración que varía con la conductividad de los materiales, pero comúnmente esta no sobrepasa los $100 \mathrm{~m}$ según (Oskooi \& Pedersen, 2005). Luego prospección EM., en esta área de estudio se llevó a cabo la ejecución de un sondeo con sistema de perforación a Rotopercusión se pudo explotar agua subterránea con un caudal de 20.000 litros/día medidos in situ.

\section{CONCLUSIONES}

- Para determinación de las posibilidades hidrogeológicas de los materiales que componen el área de investigación en el Municipio de Casas del Puerto, Ciudad de Ávila, la cual tiene como parcelas (1-18-31), los polígonos (3-4-6), con un área total de 10.844 hectáreas. Se analizaron todos los parámetros electromagnéticos, la cual revelaron anomalías importantes en todas las zonas de estudio.

- La conductividad de los cuerpos se analiza mediante la interpretación de la Componente Imaginaria (Grado de Conductividad) y la Componente Real (Indicador Superficial si el cuerpo es buen o mal conductor), obteniéndose:
Zona 1. El cambio en las componentes estación $108 \mathrm{~m}$ se asocia a picos con anomalías conductoras pequeñas y un grado de conductividad entre buen y mal conductor a lo largo de los datos filtrados en fase a lo largo del Perfil VLF-1 (Figura 4).

Zona 2. El cambio en las componentes estación $165 \mathrm{~m}$ se asocia a picos pequeños de cuerpos que son malos conductores y un grado de conductividad variable a lo largo de los datos filtrados en fase a lo largo del Perfil VLF-2 (Figura 5).

Zona 3. El cambio en las componentes estación $110 \mathrm{~m}$ se asocia a picos máximos con anomalías conductoras y un grado de conductividad entre bueno y mal conductor a lo largo de los datos filtrados en fase a lo largo del Perfil VLF-3 (Figura 6).

- La caracterización litológica e hidrogeológica de la geometría del cuerpo detectado es analizado mediante la interpretación de la Componente Real de los datos filtrados $\mathrm{KH}$, así mismo indica de forma superficial si el cuerpo es buen o mal conductor, obteniéndose:

Zona 1. Se observa que la componente en fase (real), muestra cuerpos con un ancho mediano y variables, en el gráfico de datos filtrados en fase a lo largo del Perfil VLF-1, caracterizada por anomalías del 10\% de la densidad de corriente en la pseudo sección de corriente de filtrado KH. El perfil indica zonas de materiales alterados y poco favorables para la presencia de agua subterránea (Figura 4 y 7).

Zona 2., Se observa que la componente en fase (real), muestra cuerpos con un ancho mediano y variables, en el gráfico de datos filtrados en fase a lo largo del Perfil VLF-2, caracterizada por anomalías superiores al $10 \%$ de la densidad de corriente filtrado $\mathrm{KH}$, valores anómalos debido a un contrasté de una línea

\section{Perfil VLF-3}

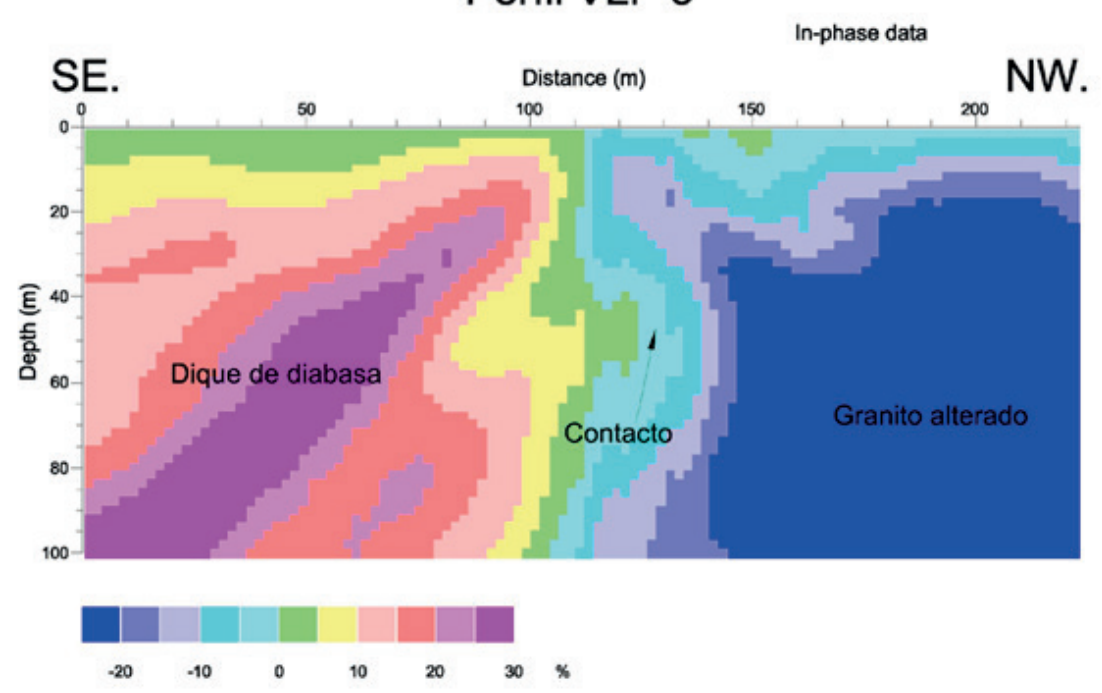

Figura 9. Sección de la parte real (en fase) de un perfil medido en la parte SW. de la ciudad de Ávila. (La zona de color celeste, verde y amarillo se asocia con porcentajes de densidad de corriente, relacionado zonas de contacto. Fuente: Elaboración propia. 
con energía eléctrica y baja posibilidad hidrológica (Figura 5 y 8 ).

Zona 3. Se observa que la componente en fase (real), muestra cuerpos con anchos definidos, en el gráfico de datos filtrados en fase a lo largo del Perfil VLF-3, caracterizada por un contrasté de anomalías del 10\% de la densidad de corriente en la pseudo sección de corriente de filtrado KH. El perfil indica zonas de contacto y alta presencia de localizar agua subterránea (Figura 6 y 9).

- Para la determinación de las zonas favorables y emplazamiento de un sondeo se debe analizar anomalías de VLF correspondiente a la curva filtrada $\mathrm{KH}$, este graficara un máximo significativo en el ángulo de inclinación y una baja anomalía en la elipticidad. La sección de densidad de corriente de filtrado KH (VLF-3), arrojó resultados asociados con los sitios donde habría las mayores posibilidades de captar aguas subterráneas del subsuelo a través de contactos geológicos y/o fracturas. Luego de las recomendaciones el pozo fue investigado mediante un sistema de perforación a Rotopercusión, dando un caudal de 20.000 litros/día medidos in situ.

\section{AGRADECIMIENTOS}

La información y el procesamiento fue realizada con el apoyo de la empresa Técnicas Geofísicas S.L.; y el Área de Prospección e Investigación Minera de la Escuela Politécnica de Ávila (Universidad de Salamanca), ÁvilaEspaña. Al Ing. Pedro Carrasco Morillo, Ing. Javier Carrasco García y al Dr. Pedro Carrasco García, profesionales que me apoyaron en los trabajos de campo y gabinete.

\section{REFERENCIAS}

ABEM Instrument AB. (1989). ABEM Printed Matter no 93057 Interpretation Guide - Theory, practice and case stories for WADI operators (p. 36). https://www.guidelinegeo.com/wpcontent/uploads/2016/03/WADI-Interpretation-guide.pdf

Adepelumi, A. A., Yi, M. J., Kim, J. H., Ako, B. D., \& Son, J. S. (2006). Integration of surface geophysical methods for fracture detection in crystalline bedrocks of southwestern Nigeria. Hydrogeology Journal, 14(7), 1284-1306. https:// doi.org/10.1007/s10040-006-0051-2

Antoniuk, J., Mościcki, J. W., Janicki, K., Górniczo -Hutnicza, A., \& Polska Miedź, K. S. (n.d.). (No Title).

Carrasco García, P. (2011). Mejora de técnicas geofisicas para la caracterización del subsuelo mediante innovación y el uso de herramientas de gestión de información espacial [Universidad de Salamanca, Ávila, España]. https://dialnet. unirioja.es/servlet/tesis?codigo $=87129$

Drahor, M. G. (2006). Integrated geophysical studies in the upper part of Sardis archaeological site, Turkey. Journal of Applied Geophysics, 59(3), 205-223. https://doi.org/10.1016/j. jappgeo.2005.10.008
GEM Systems. (2008). UAV VLF-EM SYSTEM: Resistivity Mapping Solution - Gem Systems. GSM19 Series Magnetometers V7. https:/www.gemsys.ca/uav-vlf/

Guinea, A., Playà, E., Rivero, L., Himi, M., \& Bosch, R. (2010). Geoelectrical Classification of Gypsum Rocks. Surveys in Geophysics, 31(6), 557-580. https://doi.org/10.1007/ s10712-010-9107-x

Jeng, Y., Lin, M. J., \& Chen, C. S. (2004). A very low frequencyelectromagnetic study of the geo-environmental hazardous areas in Taiwan. Environmental Geology, 46(6-7), 784-795. https://doi.org/10.1007/s00254-004-1071-7

Kaikkonen, P., \& Sharma, S. P. (1997). Delineation of nearsurface structures using VLF and VLF-R data - An insight from the joint inversion results. Leading Edge (Tulsa, OK), 16(11), 1683. https://doi.org/10.1190/1.1437559

Karous, M., \& Hjelt, S. (1983). Linear filtering of VLF dip - angle measurements. Geophysical Prospecting, 31(5), 782-794. https://doi.org/10.1111/j.1365-2478.1983.tb01085.x

Kaufman, A. A., \& Keller, G. V. (1981). The magnetotelluric sounding method. Methods in Geochemistry and Geophysics, Elsevier Scientific, Amsterdam, 15, 596. https:// doi.org/10.1016/0012-8252(83)90013-2

Kaya, M. A., Özürlan, G., \& Șengül, E. (2007). Delineation of soil and groundwater contamination using geophysical methods at a waste disposal site in Çanakkale, Turkey. Environmental Monitoring and Assessment, 135(1-3), 441-446. https://doi. org/10.1007/s10661-007-9662-x

Kobranova, V. (1990). Petrophysics. In SpringerVerlag, Berlin. INIS Repository (pp. 159-180). https://inis.iaea.org/search/searchsinglerecord. aspx?recordsFor $=$ SingleRecord $\& R N=22009434$

Oskooi, B., \& Pedersen, L. B. (2005). Comparison between VLF and RMT methods. A combined tool for mapping conductivity changes in the sedimentary cover. Journal of Applied Geophysics, 57(4), 227-241. https://doi. org/10.1016/j.jappgeo.2005.01.002

Plewa, M., \& Plewa, S. (1992). Petrofizyka. Petrofizyka. Wydawnictwa Geologiczne, - Uniwersytet Warszawski, Kraków. https://usosweb.uw.edu.pl/kontroler.php? action=actionx:katalog2/przedmioty/pokazPrzedmiot(prz kod:1300-WPFW-GES)

Sharma, S. P., \& Baranwal, V. C. (2005). Delineation of groundwater-bearing fracture zones in a hard rock area integrating very low frequency electromagnetic and resistivity data. Journal of Applied Geophysics, 57(2), 155166. https://doi.org/10.1016/j.jappgeo.2004.10.003

Spies, B. R. (1989). Depth of investigation in electromagnetic sounding methods. Geophysics, 54(7), 872-888. https://doi. org/10.1190/1.1442716

Zhdanov, M. S., \& Keller, G. V. (1994). The geoelectrical methods in geophysical exploration. The Geoelectrical Methods in Geophysical Exploration, 31. https://doi.org/10.1016/01489062(94)91092-8 